\title{
A new method of \\ presenting data referring to \\ the standard normal distribution function
}

GERALD H. FISHER

DEPARTMENT OF PSYCHOLOGY, UNIVERSITY OF NEWCASTLE UPON TYNE

Attention is drawn to certain difficulties experienced in extracting data from statistical tables cast in conventional form. A new method of representing probability values associated with the standard normal distribution function is illustrated.

If $p$ is the probability of a particular event occurring and $q$ the probability of it not doing so, then the probabilities of all possible combinations of its ocurrence and non-occurrence are indicated by the coefficients of the binomial series generated by expansion of the expression $(p+q)^{n}$. When $p=q=1 / 2$, distributions of the binomial coefficients closely resemble the normal distribution function, this being increasingly the case as the number of observations becomes large. Intuitively this might be expected, since if many independent variables are operating in a complex situation, and if it is reasonable to assume that each of these is as likely to be reflected in changes in a specified dependent variable as not, then the outcome is described by this particular case of a binomial series of probabilities. It is for this reason that quantitative measurements of a very large number of variables are found to be distributed according to the well known bell-shape of the Gaussian function.

Many statistical procedures are based upon the assumption that the data under consideration are drawn from populations which are essentially normal. Hence, it is perhaps not surprising that more than $80 \%$ of statistical procedures in common use should require reference to tables of probabilities associated with areas beneath the standard normal curve. Unfortunately, however, these and other tables do not appear to be designed to meet the needs of those whose knowledge of statistics is limited, since they appear to be both difficult to use and difficult to learn to use.

The difficulties involved may not become immediately apparent when introducing students to the use of statistical tables. Suppose that in considering the probability of occurrence of the outcome of a particular experiment, a value of Student's statistic $t$ of 1.50 has been obtained. To illustrate what is required in determination of the probability of this value having occurred by chance, or on the relevant null hypothesis, it is customary to draw the normal curve on the blackboard, erecting an ordinate at the appropriate point and subsequently extracting the value of 0.9332 from a matrix-type table referring to the normal distribution function. Using the illustration one may then continue by explaining that this value refers to the whole of the left hand area of the curve plus that part of the right hand area bounded by the ordinate erected at the value of $t$ under consideration. If the prediction has been expressed in terms of a difference in a particular direction, one then goes on to explain that the situation is known as being "onetalled," the relevant probability on the null hypothesis being $1-0.9332=0.0668$. If the prediction should not have been expressed in terms of a change in a specified direction, but simply as a difference between two samples of data obtained, then the situation is referred to as "two-tailed," in which case the appropriate probability value is $2 \times(1-0.9332)=0.1336$.

Such arithmetical operations as these are, of course, more tedious than difficult; nevertheless, students and others frequently appear to be confused in the course of carrying them out. Certain difficulties arise over exactly what the data appearing in the cells of a conventional table cast in matrix form refer to. Mistakes are made also in converting data extracted from such tables into the probability values required.

In an attempt to remove some of this confusion and to reduce the possibility of introducing errors both in extracting appropriate data and in subsequent computation, a new method of presenting probability values associated with the standard normal distribution function has been devised. This is illustrated in Fig. 1. Using this table both one- and two-tail values may be determined directly without the need for any further computation. Thus, it has the advantages of being presented in the way in which the procedures associated with it are usually taught and of reducing the number of arithmetical operations involved in determination of particular probability values. The table shown is one example of a set of statistical tables prepared by Fisher (1966), all of which are illustrated in pictorial and graphical form. Experimental studies conducted with statistical data presented in this way, in comparison with the more conventional tabular form, indicate that probability and other values can be determined many times more quickly, fewer errors being made in the course of doing so.

\section{Reference}

Fisher, G. H. The new form statistical tables. (2nd Edition). London: University of London Press, 1966. 

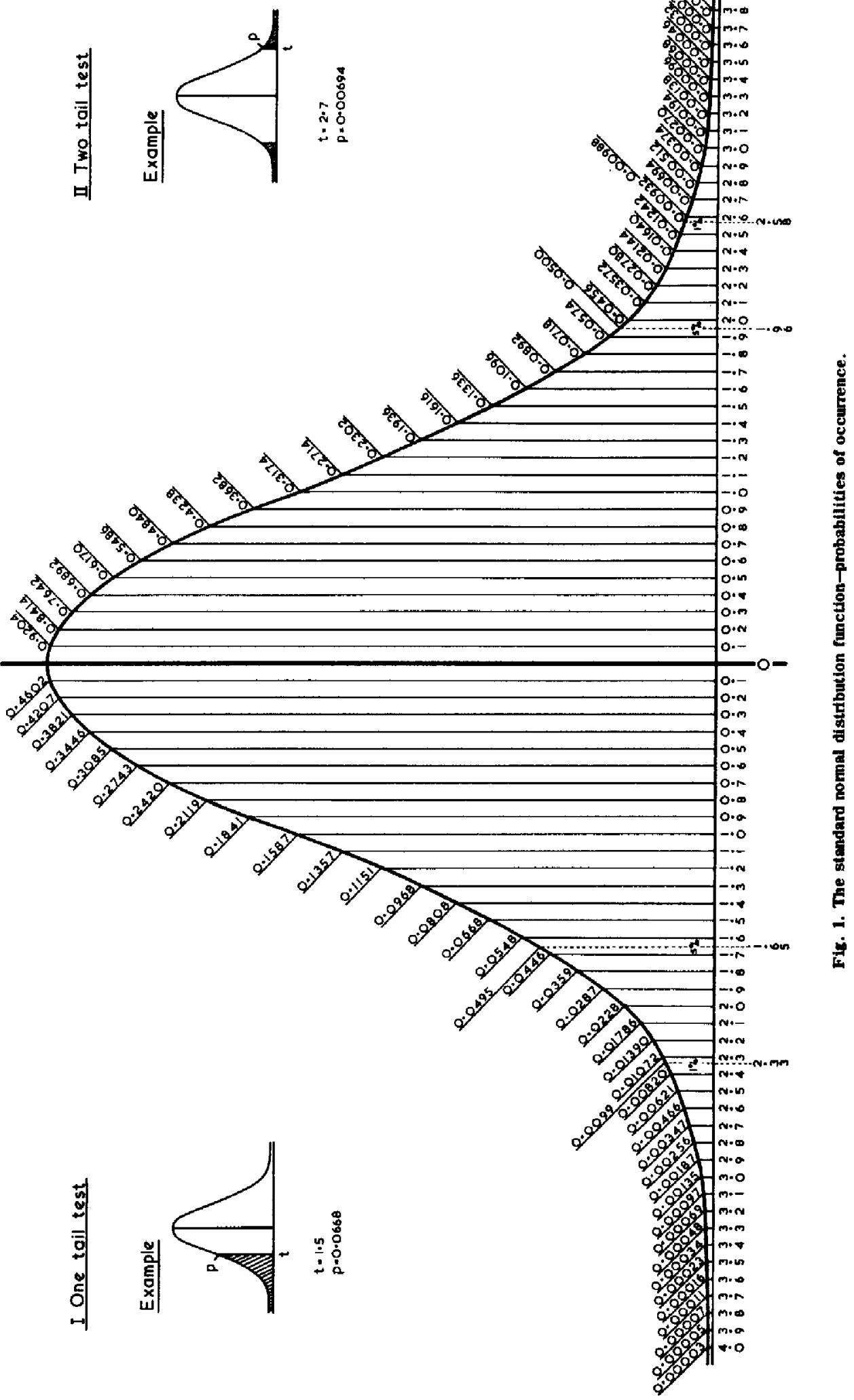